\title{
A fast method for optical simulation of flood maps of light-sharing detector modules
}

\author{
Han $\mathrm{Shi}^{1,3}$, Dong $\mathrm{Du}^{1}$, JianFeng $\mathrm{Xu}^{2}$, William W. Moses ${ }^{3}$ and Qiyu Peng ${ }^{3}$ \\ 1. Tsinghua University, Beijing, China \\ 2. Huazhong University of Science and Technology, Wuhan, China \\ 3. Lawrence Berkeley National Laboratory, CA, USA
}

\begin{abstract}
:
Optical simulation of the detector module level is highly desired for Position Emission Tomography (PET) system design. Commonly used simulation toolkits such as GATE are not efficient in the optical simulation of detector modules with complicated light-sharing configurations, where a vast amount of photons need to be tracked. We present a fast approach based on a simplified specular reflectance model and a structured light-tracking algorithm to speed up the photon tracking in detector modules constructed with polished finish and specular reflector materials. We simulated conventional block detector designs with different slotted light guide patterns using the new approach and compared the outcomes with those from GATE simulations. While the two approaches generated comparable flood maps, the new approach was more than 200 to 600 times faster. The new approach has also been validated by constructing a prototype detector and comparing the simulated flood map with the experimental flood map. The experimental flood map has nearly uniformly distributed spots similar to those in the simulated flood map. In conclusion, the new approach provides a fast and reliable simulation tool for assisting in the development of light-sharing-based detector modules with a polished surface finish and using specular reflector materials.
\end{abstract}

Index Terms: Positron Emission Tomography (PET), Block detector, Optical Simulation

\section{Introduction}

Optical simulation plays an essential role in studying Positron Emission Tomography (PET) detector performance by modeling light transport in scintillators together with light collection in photodetectors [1-3]. Optical simulation helps in understanding optical parameters that influence detector performance and assists in developing detector modules. Geant4 Application of Tomographic Emission (GATE) is a widely accepted software toolkit for conducting optical Monte Carlo simulation [4, 5].

However, popular simulation tools, including GATE, DETECT and LITRANI, are generally very slow for optical simulation task. They require considerable time and/or computation power to tracking the scintillation photons [6-8]. GATE simulation with optical process enabled is about three orders of magnitude slower than that without optical process [9]. In designing a light-sharing-based detector module, one may need to simulate and compare variants with different discrete scintillator dimensions, discrete scintillator 
array sizes, different reflectors with different lengths, different light guides, different PMT sizes, etc. Therefore, using those tools in detector module design is impractical in many situations. As a result, the design of PET detector modules depends heavily on the designers' experience and intuition and is largely based on trial and error, which is costly, time-consuming, and inefficient. PET detector modules designed this way generally do not have optimized performance. A fast approach that can accurately simulate and assist the design of optimal-performance detector modules will both speed up development and yield higher performance designs.

The quality of flood maps is one of the most essential performance factors for a light-sharing-based detector module. It is a major factor that determines the spatial resolution of a PET system. Detector modules with smaller discrete crystals and less flood map decoding error have the potential to achieve higher spatial resolutions. Many groups have simulated the light-sharing-based detector modules to assess their decoding performances using DETECT, GEANT4 and ZEMAX programs [1, 10-14]. Li and Wong has presented a mathematic model based on binomial distribution to simulate the flood map and decoding error of the Photomultiplier-Quadrant-Sharing (PQS) detectors [6, 15]. Those mathematic-model-based simulations are generally less accurate but much faster than Monte Carlo simulations. With the help of the simulation, Li was able to get to the final optimized PQS design with only a few development cycles.

The main purpose of this study is to develop a fast optical simulation method to simulate detector modules with complicated light-sharing schemes, e.g., a conventional block detector with different slotted

light guide patterns. In this paper we describe our method, which is based on a specular reflectance model and a structured light-tracking algorithm, and apply it in the flood map simulations of two typical block detector designs. GATE simulations with similar settings are performed to validate the new method. As an example application, we demonstrate how to use our method to optimize the slotted light guide pattern design for a conventional detector module. A prototype detector was constructed to validate the simulation-based optimization method. Note that the simulation tool developed in this study is based on specular reflectance model, and it is only for detector modules with a polished surface finish and using specular reflector materials.

\section{Methods}

\subsection{Detector module geometries}

Two typical light-sharing-based block detector modules as shown in Fig. 1 are simulated in this study. The first module (Fig. 1a) is a conventional block detector consisting of a layer of a $12 \times 12$ discrete scintillator array, a light guide and four round PMTs. Reflectors are attached to five surfaces of each 
scintillator crystal, except for the surface coupled to the light guide. The four outer sides of the light guide are wrapped with light reflectors. The top side of the light guide is coupled with the discrete scintillator array, and the bottom side is coupled with the four PMTs. The diameter of each PMT is equal to half the width of the crystal array. The effects of the optical properties of coupling materials, such the index of refraction and attenuation length, are not considered in this study.

The second design (Fig. 1b) is similar to the first one, except that its light guide has slots with specular reflectors inserted. Twenty-two reflectors with different lengths are symmetrically distributed in the 22 light guide slots (11 in the x direction and 11 in the y direction). The 22 reflectors have only six different lengths due to the geometric symmetry of the detector module.

The detailed dimensional settings of the two detector modules, including the sizes of the discrete scintillators, light guide and PMTs are summarized in section 2.2.7.

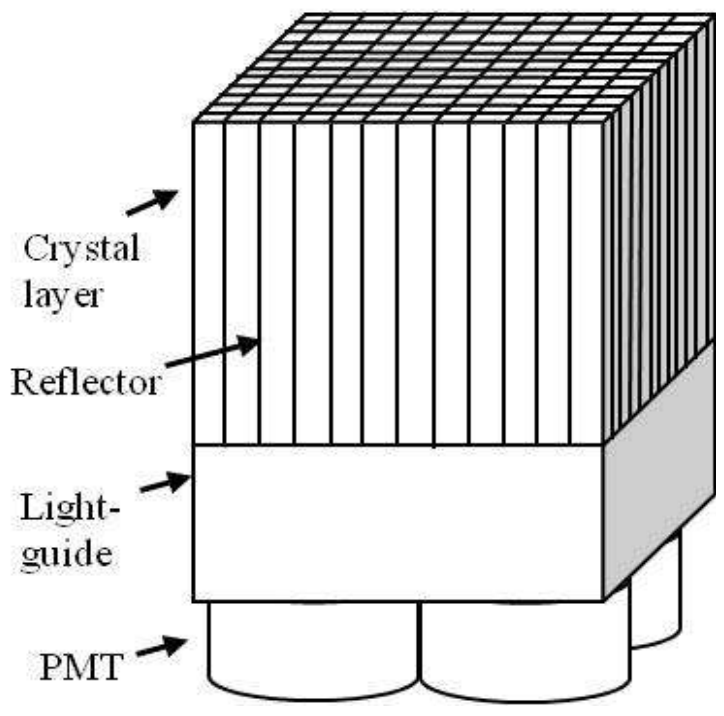

(a)

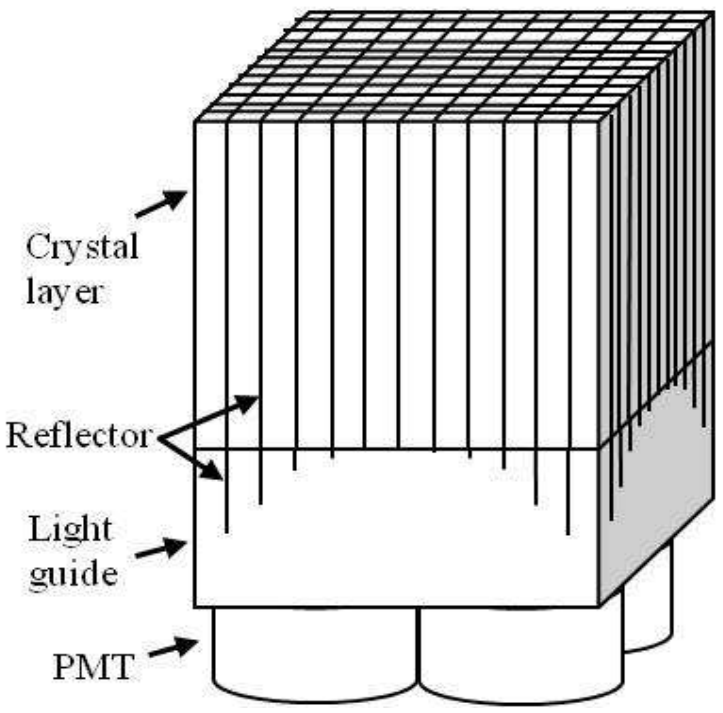

(b)

Fig. 1. The detector modules (a) without and (b) with slots in the light guide.

\subsection{Simulation methods}

\subsubsection{Simulation of the optical transport procedure}

The optical simulation method includes a box-shaped specular reflectance model (where we define a "box" to be a rectangular parallelpiped) and a structured algorithm to track photons traveling from box to box. As shown in Fig. 2, the $\gamma$ photons interact at a randomly chosen position in the discrete scintillator array, and emit all of their scintillating photons from this point (i.e., neither Compton interactions nor x-ray 
fluorescence are simulated). This position is uniformly distributed in both $\mathrm{x}$ and $\mathrm{y}$, but follows an exponential attenuation law (with appropriate attenuation length) in depth.

The scintillating photons bounce back and forth in the box-shaped scintillators with five surfaces wrapped with specular reflectors until they are absorbed in the scintillators or exit through the surface that is coupled with the light guide. The photons undergo reflection or refraction on the scintillator to light guide interface, which will be further addressed in section 2.2.4. The photons entering the light guide bounce back and forth in a virtual box in the light guide with four surfaces mounted with specular reflectors until they are absorbed or exit through the lower surface of that virtual box and enter the next one in the light guide. Except for the absorbed portion, the unabsorbed photons entering from the upper surface of the virtual box cannot travel back to the upper surface and can exit only through the lower surface of that box. The procedure is repeated until the photons are absorbed in the light guide, exit the light guide and enter the PMTs, or get lost in the gaps between the PMTs.

The locations and three-dimensional sizes of the virtual boxes are calculated and saved in a Look-Up Table (LUT) according to the detector module settings before the simulation starts. 


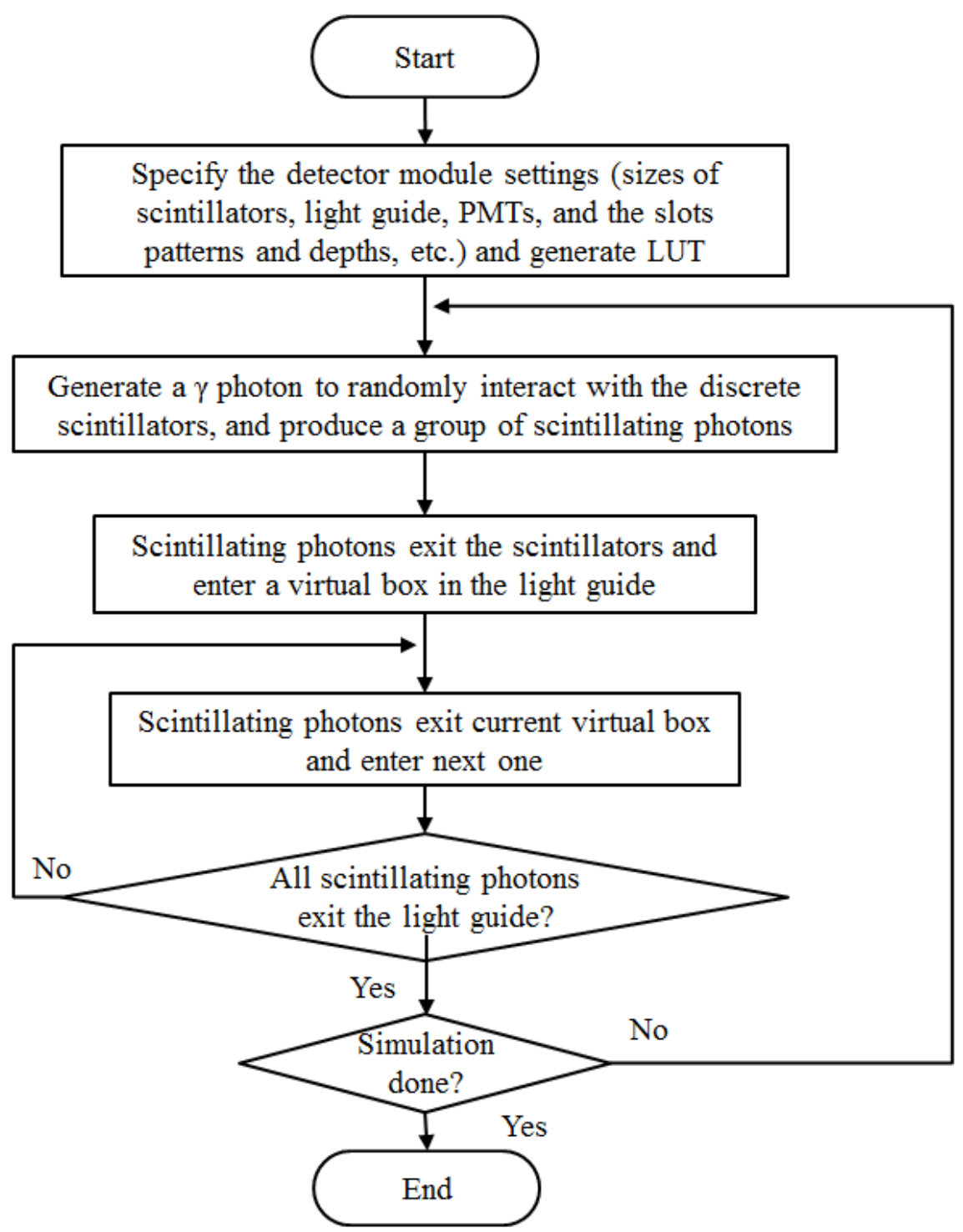

Fig. 2. Flow chart of the optical simulation method.

\subsubsection{Box-shaped specular reflectance model}

We use a box-shaped specular reflectance model to simplify and speed up the light-tracking computation. This model has two main assumptions: 1) all reflections in the module are specular (i.e., the angle of incidence equals the angle of reflection), and 2) all the optical elements (the scintillator crystals and light guide) are built up from rectangular parallelpiped elements that we refer to as "virtual boxes. Using this model, the exiting positions and directions of the scintillating photons from a virtual box can be computed easily from their entering positions and directions.

The specular reflectance model accurately mimics detector module designs with a polished surface finish and using specular reflector materials such as Enhanced Specular Reflector (ESR) film. M. Janecek's optical reflectance measurement studies show that the specular reflection peak at different incident angles has a constant intensity at the reflective angle equal to the incident angle for ESR film [16-18]. To verify that, we 
also simulated the two detector modules using GATE with the UNIFIED model. The simulation results of two different models are compared in section 3.1 and 3.2.

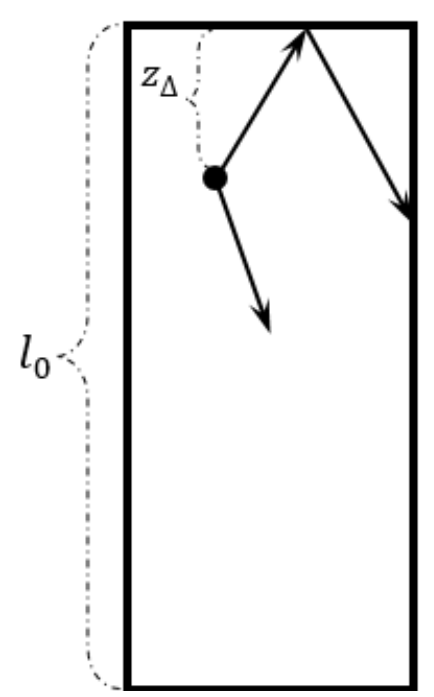

(a)

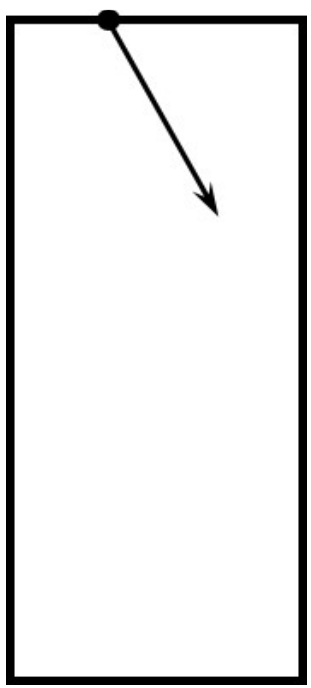

(b)

- $\left(x_{i n}, y_{i n}, z_{i n}\right),\left(V_{x_{-} i n}, V_{y_{-} i n}, V_{z_{-} i n}\right)$

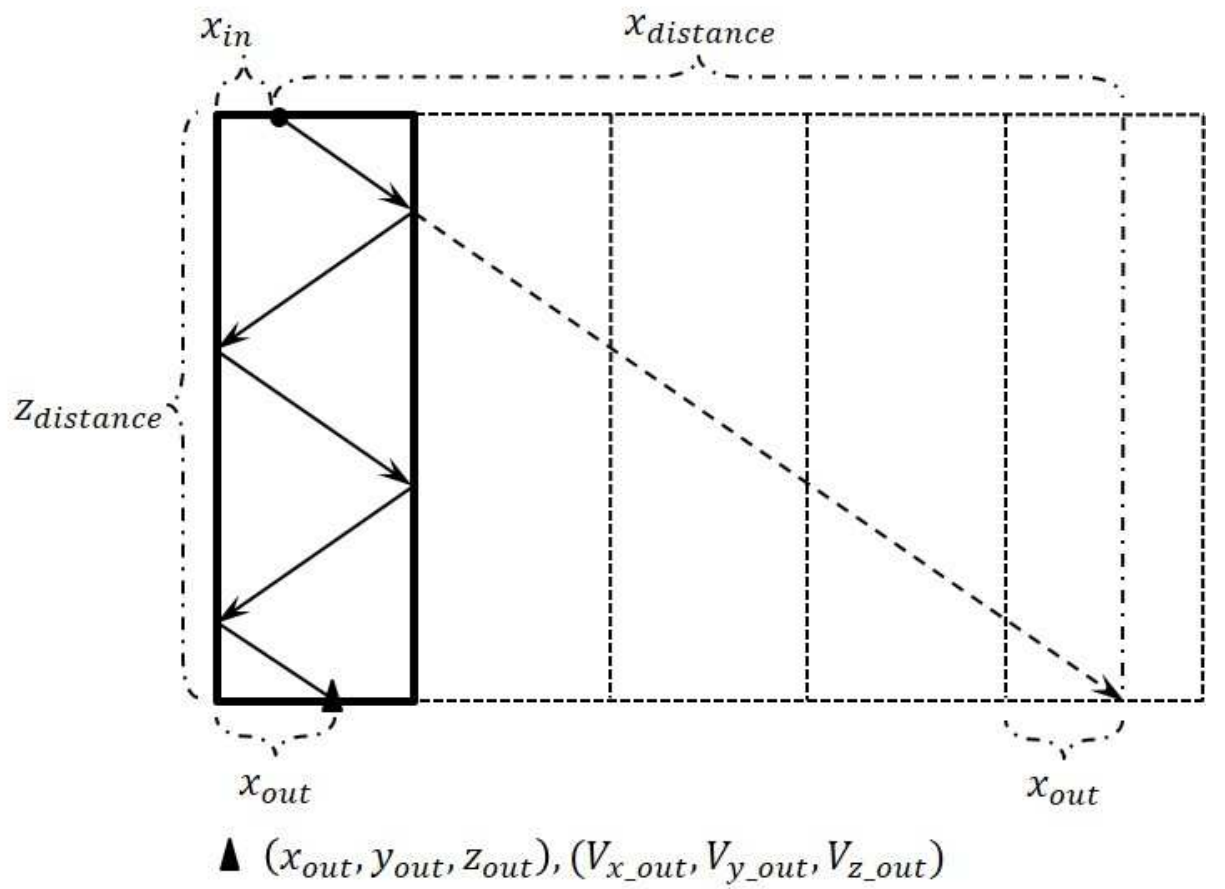

(c)

Fig. 3. Illustration of the photon transmission (a) in crystal and (b) in light guide. (c) Photon tracking in light guide. The positions where a photon enters and exits from the virtual box are marked by the solid dot and the solid triangle respectively. The solid arrows are the photon trajectories, and the dashed lines are auxiliary to demonstrate the geometric symmetry applied to facilitate the calculation. Similar method is used to track photons in the crystal. 
There are two situations when box-shape model is used to tracking the photons: in crystal and in light guide, as shown in Fig. 3a and Fig. 3b respectively. Fig. 3c demonstrates how the geometric symmetry is used to facilitate the photon tracking in the light guide. The positions and directions of the photons travelling out of the box can be computed directly from the positions and directions of input photons. While implementing the algorithm, we define a function whose inputs are the three side lengths of the virtual box $\left(l_{x}, l_{y}, l_{z}\right)$, as well as the incident photon position and direction. Given specular reflectance, there is no need to explicitly simulate every reflection, which speeds up the photon tracking procedure. The function is called to calculate the exiting position $\left(x_{\text {out }}, y_{\text {out }}, z_{\text {out }}\right)$ and direction $\left(V_{x_{-} \text {out }}, V_{y_{-} \text {out }}, V_{z_{-} \text {out }}\right)$ rapidly every time when a photon enters a virtual box from a position $\left(x_{i n}, y_{i n}, z_{i n}\right)$ and direction $\left(V_{x_{-} \text {in }}, V_{y_{-} i n}, V_{z_{-} i n}\right)$ :

$$
\begin{gathered}
\left\{\begin{array}{c}
x_{\text {out }}=\left(x_{\text {distance }}+x_{\text {in }}\right) \bmod l_{x} \\
y_{\text {out }}=\left(y_{\text {distance }}+y_{\text {in }}\right) \bmod l_{y} \\
z_{\text {out }}=z_{\text {in }}+l_{z}
\end{array}\right. \\
\left\{\begin{array}{c}
V_{x_{\_} \text {out }}=V_{x_{\_} \text {in }} \times\left(1-2 \times\left(N_{x} \bmod 2\right)\right) \\
V_{y_{-} \text {out }}=V_{y_{-} \text {in }} \times\left(1-2 \times\left(N_{y} \bmod 2\right)\right) \\
V_{z_{-} \text {out }}=V_{z_{-} \text {in }}
\end{array}\right.
\end{gathered}
$$

where mod represents for the modulo operation. $x_{\text {distance }}, y_{\text {distance }}$ and $z_{\text {distance }}$ are

$$
\left\{\begin{array}{c}
x_{\text {distance }}=l_{z} / V_{z_{\_} i n} \times V_{x_{\_} \text {in }} \\
y_{\text {distance }}=l_{z} / V_{z_{\_} i n} \times V_{y_{\_} i n} \\
z_{\text {distance }}=l_{z}
\end{array}\right.
$$

The distance of flight $D$ and times of reflection $N$ in each virtual box is also calculated conveniently:

$$
\begin{aligned}
D & =\sqrt{x_{\text {distance }}{ }^{2}+y_{\text {distance }}{ }^{2}+z_{\text {distance }}{ }^{2}} \\
N & =\operatorname{fix}\left[\left(x_{\text {distance }}+x_{\text {in }}\right) / l_{x}\right]+\operatorname{fix}\left[\left(y_{\text {distance }}+y_{\text {in }}\right) / l_{y}\right]
\end{aligned}
$$

where fix $(x)$ is a function that rounds $x$ to the nearest integer (towards zero).

We assume that the photons cannot penetrate the reflectors. The possibility of a photon exiting from a virtual box without being absorbed is derived directly from this simple equation:

$$
P=R_{e}^{N} \times e^{-D / \mu}
$$

where $R_{e}$ and $\mu$ are the reflective coefficient of the specular reflector and attenuation length of the scintillator or light guide, respectively.

Photons tracking in crystal are implemented conveniently by setting the lengths of the virtual box to $l_{z}=l_{0}-z_{\Delta}\left(\right.$ or $\left.l_{z}=l_{0}+2 z_{\Delta}\right)$ for the photons moving down (or up). $z_{\Delta}$ is the location of the scintillating photon in z-direction in the crystal (shown in Fig. 3 a).

\subsubsection{Structured light-tracking algorithm}

Tracking scintillating photons in detectors without slots in the light guide is quite simple. All the scintillating photons, e.g., photons from positions A and B shown in Fig. 4 a, have similar transport 
procedures. They are generated in discrete scintillators, enter the same virtual box that is essentially the whole light guide, and are absorbed or exit from the light guide to be detected by PMTs or get lost in the PMT gaps.

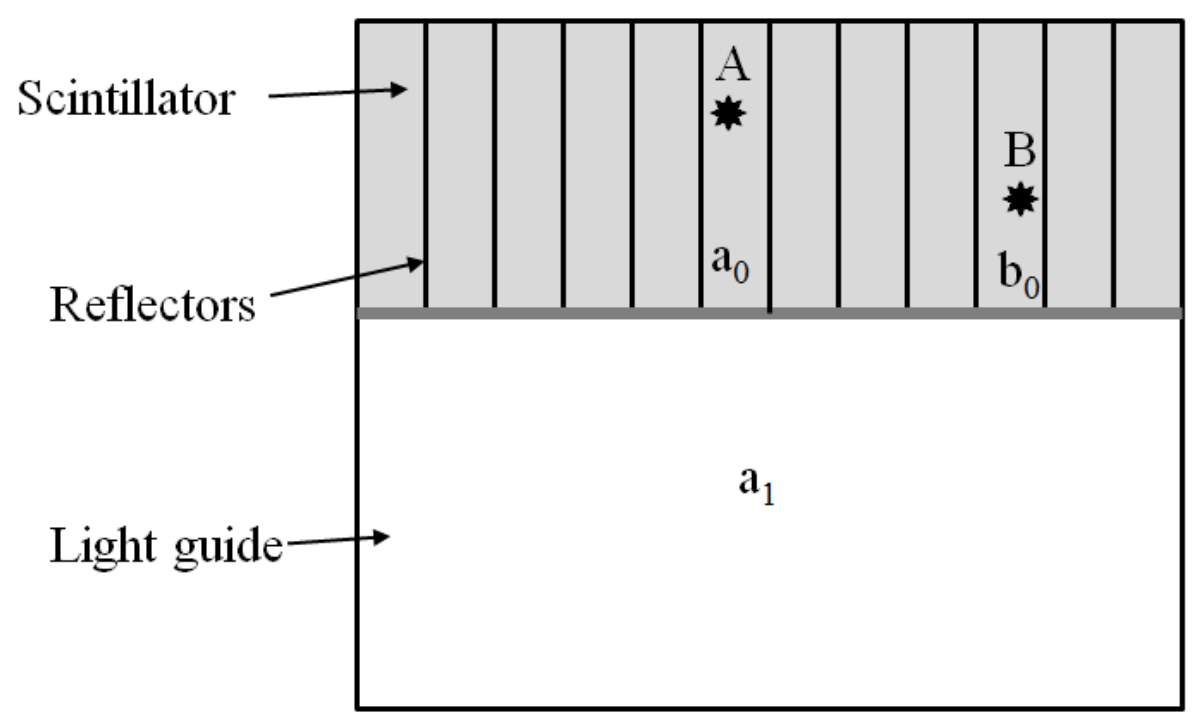

(a)

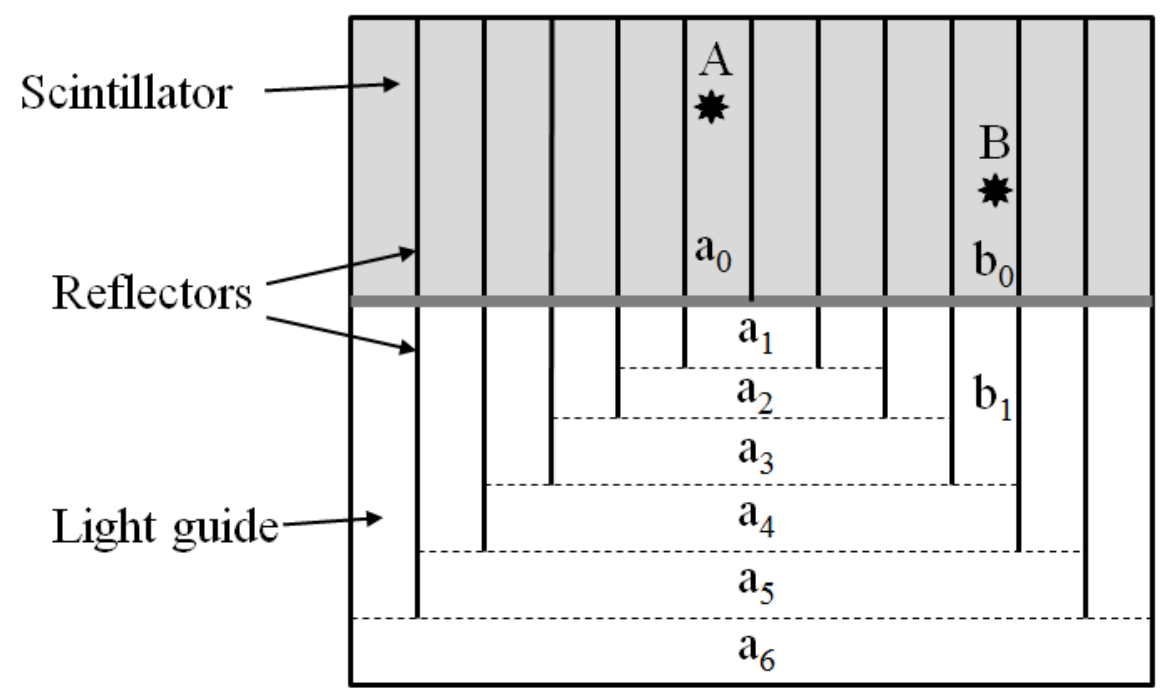

(b)

Fig. 4. Illustrations of the light tracking in structured virtual boxes. Detectors (a) with and (b) without slots in the light guide.

Tracking scintillating photons in detectors with slotted patterns in the light guide is more complicated. Fig. $4 \mathrm{~b}$ illustrates photon tracking in a detector with the one-dimensional symmetric slotted patterns. The scintillating photons from positions A and B have very different routes when they travel through the virtual boxes. Photon A travels through seven virtual boxes (a0, a1, a2, a3, a4, a5, and a6) before exiting from the light guide. Photon B travels through five virtual boxes (b0, b1, a4, a5, and a6) before exiting from the light guide. The situations get more complicated when simulating a detector with less symmetry. 
The boundaries of virtual boxes and the virtual-box sequences of the scintillating photons generated in all discrete scintillators are calculated and saved in a Look-up Table (LUT) before the simulation starts. During the simulation, the virtual-box sequence of a photon is read from the LUT. The photon sequentially travels though the virtual boxes. The exiting positions and directions of the photon from the virtual boxes are calculated using the method described in section 2.2.2.

\subsubsection{Photon transmission and reflection between crystal-light guide interface}

The crystal and light guide can have different refractive indexes. The photon transport follows the Fresnel equations on the crystal-light guide interface. Total internal reflection occurs when the incident angle of a photon is larger than the critical angle. The photons could bounce back into the crystal and specularly reflected by the other five sides attached with reflectors. In the specular reflectance model, the incident angles do not change when those photons bounced back and reach the crystal-light guide interface again. Thus, those photons are all absorbed in the crystal or by the reflectors after many times total reflection. When the incident angle of a photon is smaller than the critical angle, it can be reflected or refracted. The chances of reflection or refraction are calculated using Fresnel equations. The reflected photons should be tracked again until they are absorbed or enter the light guide. However, more than $99.9 \%$ of the photons are absorbed or exit from the crystal before being reflected three times on the crystal-light guide interface when indexes of refraction of the crystal and light guide are $n=1.82$ and $n=1.42$ respectively. To speed up the computation, the photons are considered absorbed when they are reflected more than three times on the interface in the simulation.

\subsubsection{Flood map and crystal decoding errors}

Anger-logic positioning based on light sharing is used to calculate the flood map:

$$
\begin{aligned}
& X=\frac{(A+B)-(C+D)}{A+B+C+D} \\
& Y=\frac{(A+C)-(B+D)}{A+B+C+D}
\end{aligned}
$$

where A, B, C and D are the signal magnitude (number of photons) detected by the four PMTs respectively in each interaction as shown in Fig. 5. 


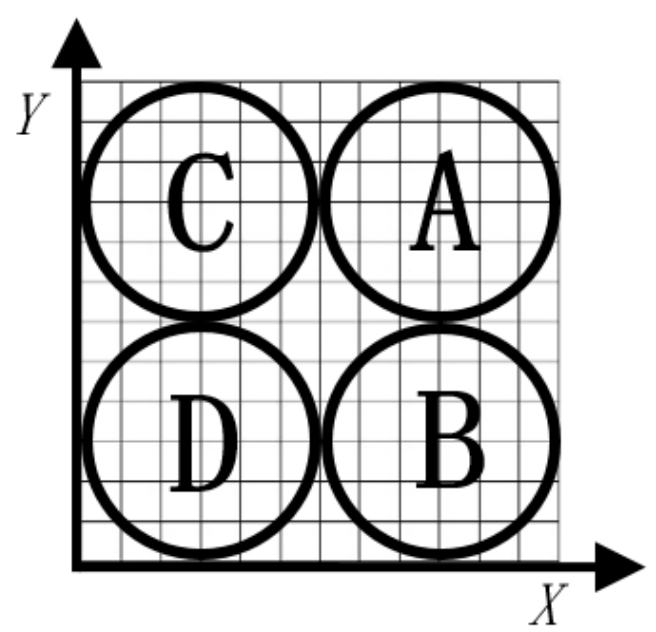

Fig. 5. Anger-logic positioning for light-sharing block detector

As shown in Fig. 6, we evaluate the quality of the flood map using the FWHM of the crystal spot $\left(F W H M_{x}\right.$ and $\left.F W H M_{y}\right)$, the distances between adjacent spots $\left(D_{x}\right.$ and $\left.D_{y}\right)$, and the ratio of the distances to the FWHMs:

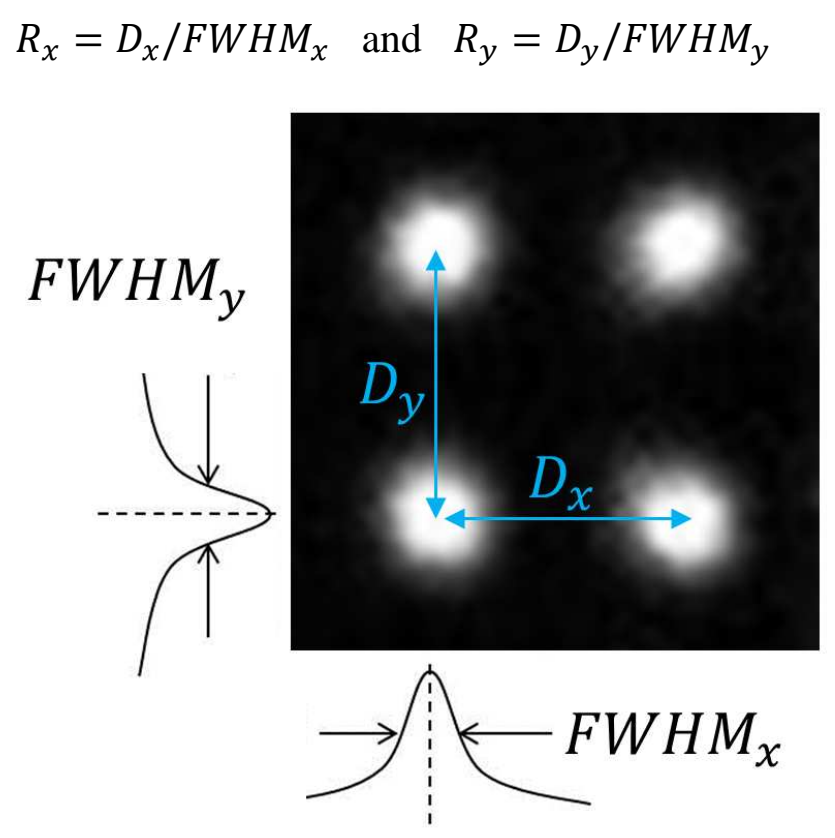

Fig. 6. The quality of the flood map is evaluated by FWHM of the crystal spot, the distances between adjacent spots, and the ratio of the distances to the FWHMs.

We segmented the flood maps by drawing horizontal and vertical lines midway between adjacent peaks in the flood maps. Then, we calculated the ratio of the total number of the misdecoded events to the total event number (the Overall Decoding Error Rate, ODER) for each flood maps. Precise evaluation of the decoding errors for individual discrete crystals depends not only on the quality of the flood map, but also on 
the flood map segmentation algorithm. Identifying the optimal segmentation algorithm is beyond the scope of this paper $[19,20]$.

\subsubsection{Optimization of the slot depths}

In a light-sharing-based detector module, the slotted light guide is essential for achieving a good discrete crystal decoding performance [2, 6, 21]. A well-designed light guide with optimized slot depths can efficiently alter the photon distributions among the four PMTs and minimize the overlap between discrete crystal spots in the flood map. However, optimizing the slot depths is a difficult task. Many experiments need to be performed to tune the slot depths before one can finalize a light-sharing-based detector module design scheme.

Our simulation method can be applied to optimize the slot depth design for a conventional detector module. Only six different lengths $\left(a_{0} \sim a_{5}\right)$ need to be optimized due to the geometric symmetry of the block detector. The optimization procedure is demonstrated in Fig. 7. In the simulation, we first tune the depth of slot $a_{0}$ to ensure that the spot in the flood map of discrete crystal $A_{0}$ is aligned with the center of the physical crystal. Next, we tune the depth of slot $a_{1}$ to ensure that the spot in the flood map of discrete crystal $A_{1}$ is aligned with the center of the physical crystal. The procedure is repeated until the depths of all the slots are determined. This algorithm is based on the assumption that the spot position of the discrete crystal $A_{0}$ doesn't change much when we tune slot $a_{1} \sim \operatorname{slot} a_{5}$. That assumption is generally true, provided that slot lengths $a_{0}>a_{1}>\cdots>a_{5}$. Also, note that the slot lengths are optimized by arranging the spots of discrete crystals uniformly in the flood map. It is a reasonable strategy to decrease the decoding errors by uniformly distributing the crystal spot in the flood map, although this algorithm does not guarantee the minimized decoding error. 


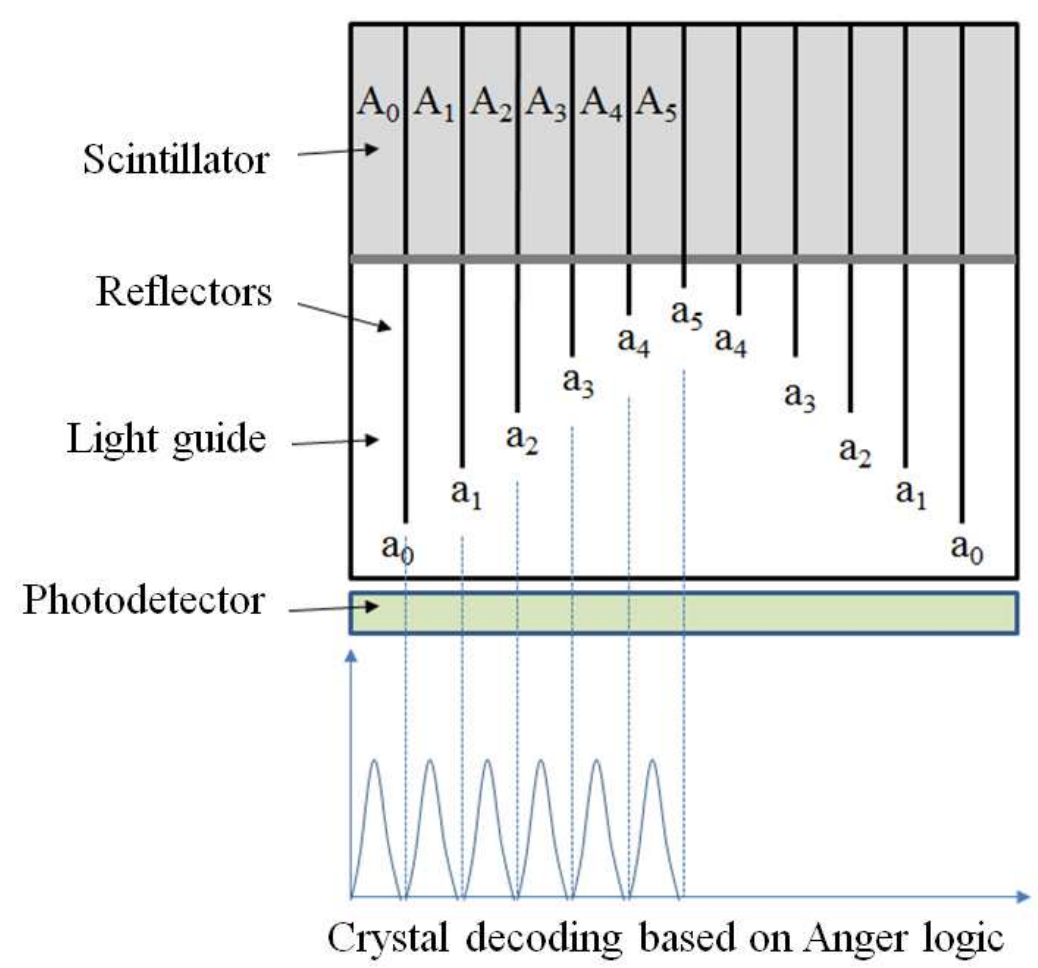

Fig. 7. Illustration of the procedures of the slot depths calculation.

\subsubsection{Simulation parameters and settings}

The fast simulation algorithm based on the simplified specular reflectance model and the structured light-tracking algorithm is implemented in Matlab R2013b. The basic parameters used in simulation are listed in Table 1. The scintillator is LYSO with a light yield of 26,000 photons per MeV and $0.87 \mathrm{~cm}-1$ linear attenuation coefficient [22]. $511 \mathrm{keV}$ gammas with constant light output are used in the simulations. The attenuation length of $420 \mathrm{~nm}$ photons in both LYSO and the light guide is $42 \mathrm{~cm}$ [23]. The effects of the coupling materials are not considered. The reflectors are ESR film with the reflective coefficient of 0.985 [18]. The photon detection efficiency of the PMT is $25 \%$.

There are 144 discrete crystals in the $12 \times 12$ array. The discrete crystal size is $4 \mathrm{~mm} \times 4 \mathrm{~mm} \times 25 \mathrm{~mm}$. The thickness of the reflector is $0.15 \mathrm{~mm}$. The size of the light guide is $49.65 \mathrm{~mm} \times 49.65 \mathrm{~mm} \times 16 \mathrm{~mm}$. The gap in the light guide is $0.15 \mathrm{~mm}$ thick, where the reflector is fully filled.

The same detectors are also simulated using GATE with the UNIFIED model. In the GATE simulation, the reflection type is set to $100 \%$ specular lobe distribution. The surface type is set to dielectric metal to simulate the ESR film. The surface finish is set to polished with the deviation of reflective angle equal to $0.31^{\circ}$, which was measured by Janecek with a Dektak 150 surface profilometer on a mechanically polished surface [18]. Other parameters used in GATE simulation are summarized in table 1. A Linux shell program was developed to call GATE macro programs and Octave programs to generate and analyze GATE simulation data. 
Table 1. Parameters used in both the Matlab and GATE simulations

\begin{tabular}{|l|l|}
\hline Scintillation material & LYSO \\
\hline Light yield & 26,000 photons per MeV \\
\hline Linear attenuation coefficient & $0.87 \mathrm{~cm}^{-1} @ 511 \mathrm{keV}$ \\
\hline Visible light attenuation length & $42 \mathrm{~cm}$ \\
\hline Refractive index & 1.82 \\
\hline Reflector thickness & $0.15 \mathrm{~mm}$ \\
\hline Reflective coefficient & 0.985 \\
\hline Light guide attenuation length & $42 \mathrm{~cm}$ \\
\hline Light guide refractive index & 1.42 \\
\hline PMT photon detection efficiency & $25 \%$ \\
\hline
\end{tabular}

\subsection{Experimental methods}

A prototype detector was constructed with optimized slot depths to validate both the simulation and optimization methods. The prototype detector was constructed using a liquid light guide (silicone oil) and a metal grid-shape framework shown as Fig. 8.26 pieces of thin metal slices glued with reflectors were used to build the metal grid-shape framework. The discrete crystals were inserted into the metal grid-shape framework. The metal grid-shape framework with crystal array was then submerged in silicone oil (shown as blank area in the dashed box). The silicone oil layer between the scintillators and the PMTs acted as the liquid light guide. The prototype detector consisted of a metal framework with different reflector heights, a $12 \times 12$ array of discrete LYSO scintillators and four Hamamatsu R9800 PMTs. The thickness of the metal slices was $0.05 \mathrm{~mm}$, and the width was $52 \mathrm{~mm}$. The thickness of the ESR reflectors was about $0.07 \mathrm{~mm}$. The discrete LYSO scintillators were $4 \mathrm{~mm} \times 4 \mathrm{~mm} \times 25 \mathrm{~mm}$. The diameter of the PMTs was $25.4 \mathrm{~mm}$. The size of the 12 $\times 12$ scintillator array was $50.4 \mathrm{~mm} \times 50.4 \mathrm{~mm} \times 25 \mathrm{~mm}$. The prototype detector was characterized using a

${ }^{22} \mathrm{Na}$ source. The analog signals from 4 PMTs were recorded to calculate the flood map using Anger-logic.

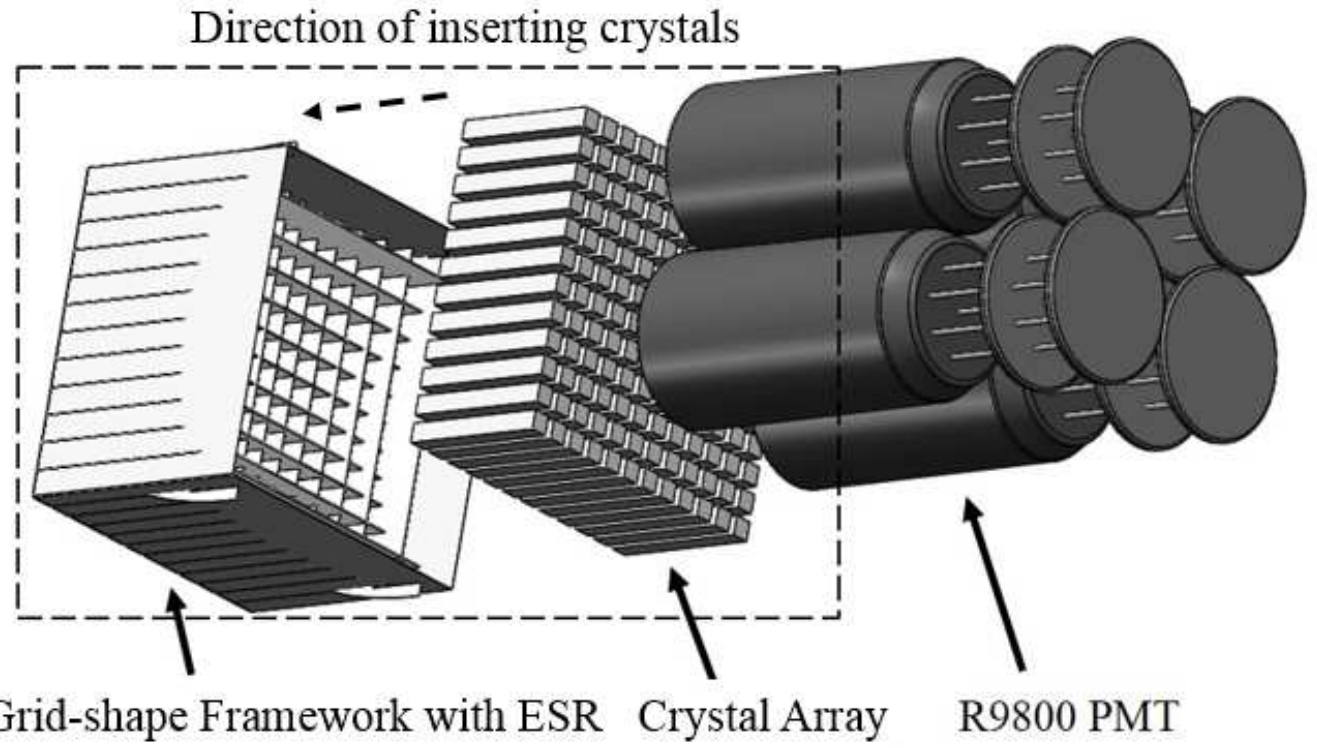

Fig. 8 Illustration of the prototype detector. The crystal array was inserted to the metal grid-shape framework (shown by the dashed arrow in the figure). The gap between the crystal array and the PMTs was filled with silicone oil. 


\section{Results}

\subsection{Single crystal light distribution}

Single crystal light distributions are calculated to compare the simplified specular reflectance model with the UNIFIED model used in GATE [22]. In the simulation, a single crystal (4 $\mathrm{mm} \times 4 \mathrm{~mm} \times 25 \mathrm{~mm}$ in size) sits on a large light guide $(200 \mathrm{~mm} \times 200 \mathrm{~mm} \times 16 \mathrm{~mm})$. Light distributions are calculated using both models. Fig. 9 compares the profiles of the light distributions. There are no significant differences between the two profiles. The FWHMs of the two curves are $14.5 \mathrm{~mm}$ (the simplified specular reflectance model) and 14.3 $\mathrm{mm}$ (the UNIFIED model) respectively. This is to be expected, and in fact, lack of agreement would indicate a problem, as the optical properties (reflectance, absorption, geometry, etc.) simulated are similar in the two simulations.

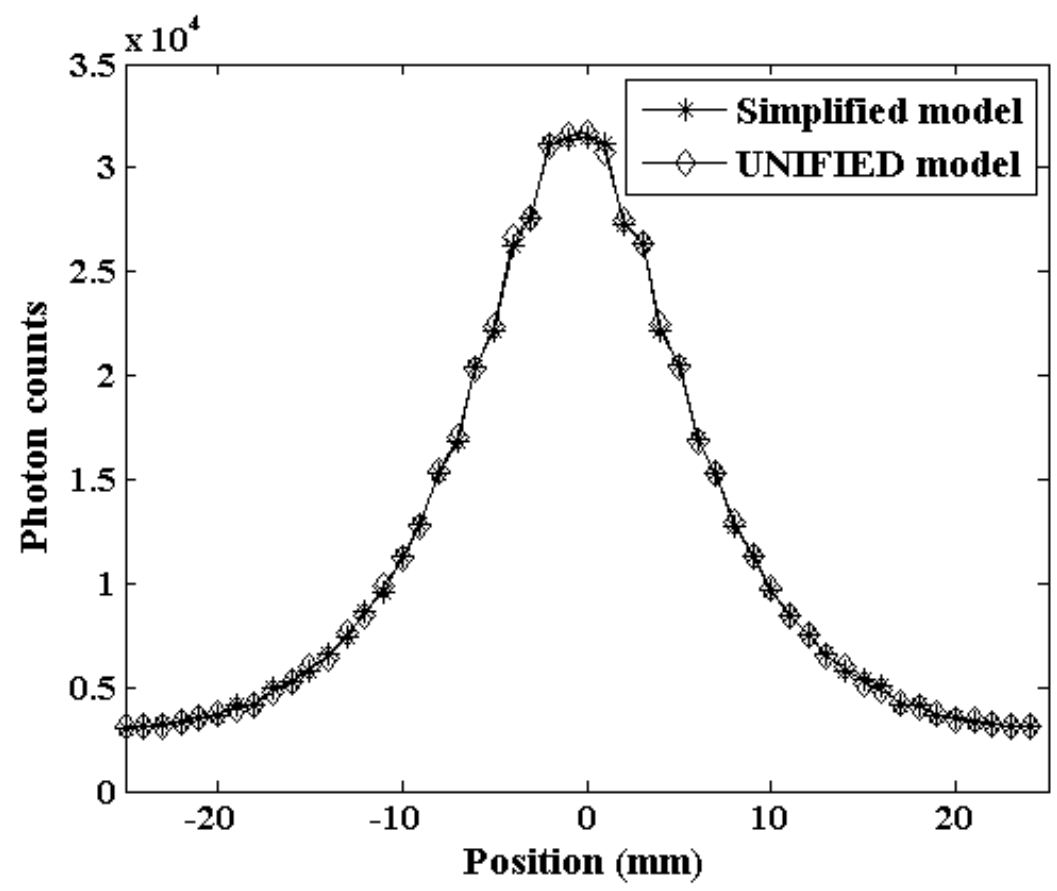

Fig. 9. Comparison of the light distribution profiles of the simplified specular reflectance model and the UNIFIED model.

\subsection{Flood map}

Fig. 10 shows the simulation results of the detector modules with a $16 \mathrm{~mm}$ unslotted light guide (Fig. 1a). The results show that the flood maps generated by the simplified specular reflectance model and by the UNIFIED model are very similar. Again, the results should have very close agreement as the optical properties simulated are very similar. The simulation times for the two models are 788 seconds and 163,200 seconds (45.3 hours) respectively. Fig. 11 compares the two flood maps quantitatively in terms of differences in spot positions (centers of the spots) and spot sizes (averaged FWHMs in $\mathrm{x}$ and $\mathrm{y}$ direction for each spot). 
The resolutions of the flood maps are $256 \times 256$ pixels. The average and the maximum differences in spot positions ( 0.9 pixels and 1.5 pixels respectively in the $256 \times 256$ pixel flood maps $)$ and sizes $(0.1$ pixels and 2.0 pixels respectively) are very small. The differences in center locations are the distances between the center positions of the spots. We didn't consider the directions of the distances in the calculation. Thus, the average of the differences of the center locations is not zero. The average spot size of the flood map generated using the UNIFIED model (7.8 pixels) is slightly smaller than that generated using the simplified specular reflectance model (7.9 pixels). There are no significant differences between the flood maps generated by two models.

The average numbers of photons getting collected by 4 PMTs are about 740 and 742 for the simplified simulation and GATE simulation respectively. The ratio of number of photons collected by PMTs to number of photons produced by gamma interaction is about 5.6\%. In our simulations, the light collection efficiency of the PMT is set to $25 \%$. The filling factor of the $2 \times 2$ PMT array is $78.5 \%$. Because of the total reflection, only $37.4 \%$ photons are able to penetrate the interface between the crystal and the light guide. There are $24.3 \%$ extra photons loss caused by the absorption of the reflector and the light attenuation in the crystal and light guide. Thus, the total light collection ratio is about $5.6 \%$.

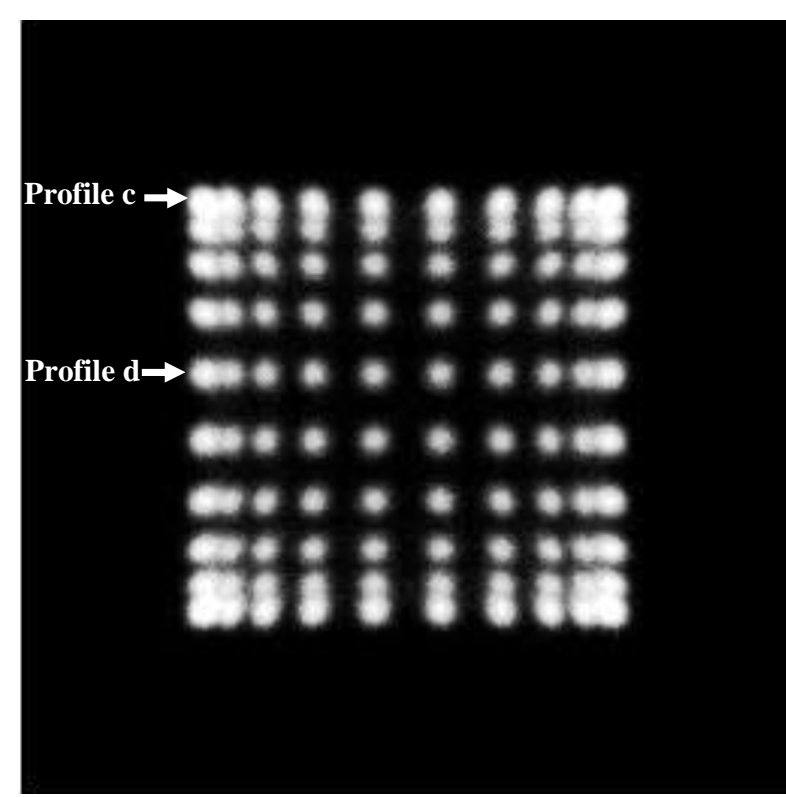

(a)

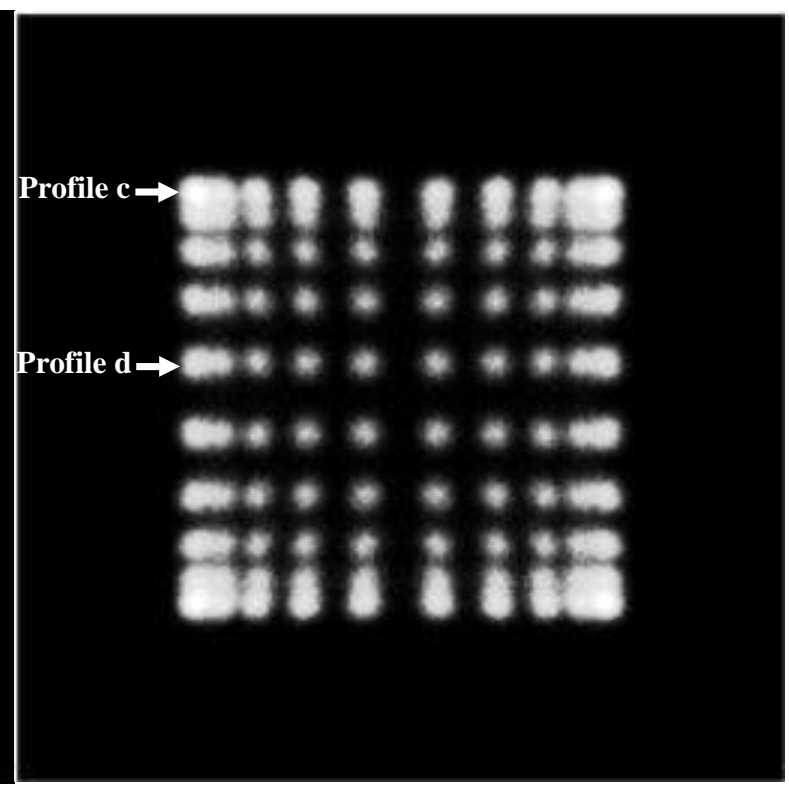

(b) 


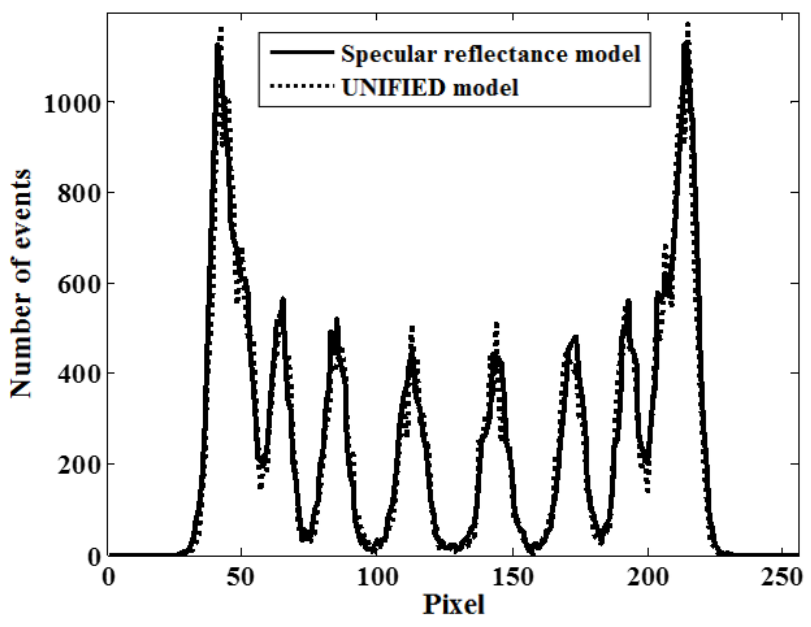

(c)

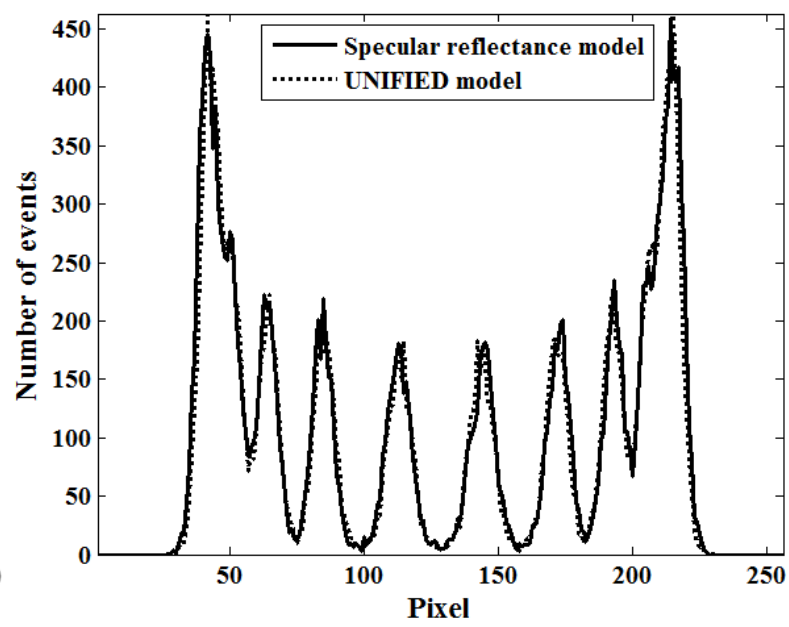

(d)

Fig. 10. Flood maps of a $12 \times 12$ detector block coupled with a $16 \mathrm{~mm}$ unslotted light guide. The resolutions of the flood maps are $256 \times 256$ pixels. The two flood maps are generated using (a) the simplified specular reflectance model and (b) the UNIFIED model respectively. Profiles through (c) the top line and (d) the center line marked in the flood maps. The solid curve and the dotted curve are profiles from the simplified specular reflectance model and the UNIFIED model respectively. The profiles from the two flood maps are very similar.

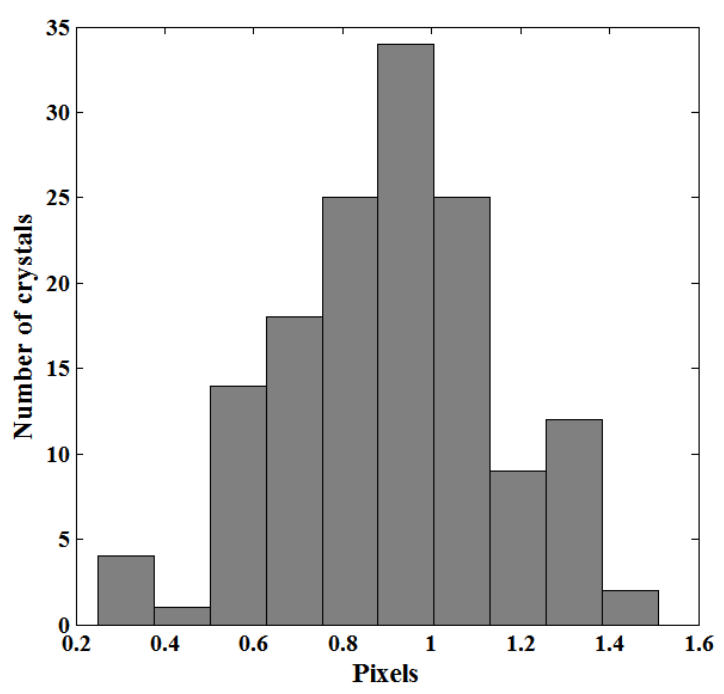

(a)

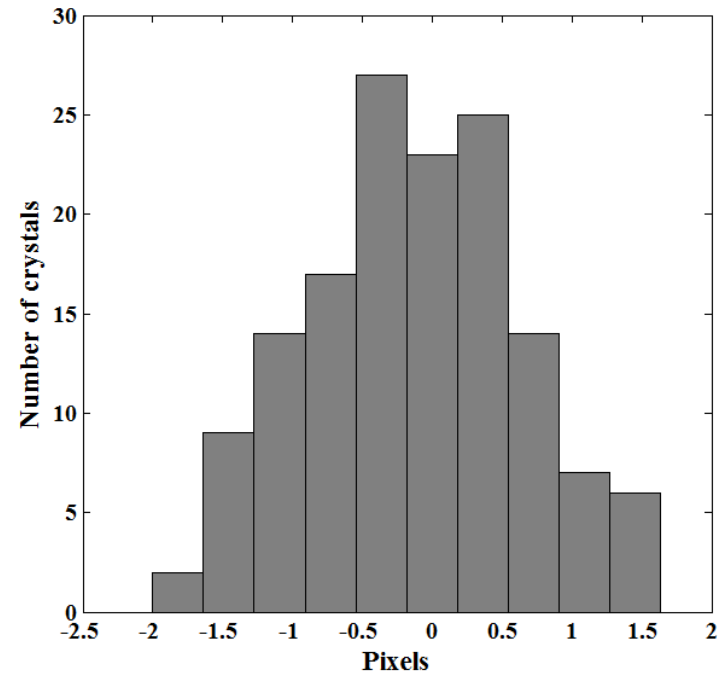

(b)

Fig. 11. The distributions of the differences of (a) the center locations and (b) the FWHMs of the 144 crystal spots of the two flood maps shown in Fig. 10. The average and the maximum differences of the center locations are 0.9 pixels and 1.5 pixels respectively. The average and the maximum differences of the FWHMs are 0.1 pixels and 2.0 pixels respectively.

Fig. 12 shows the simulation results of the detector modules with slots in the light guide (Fig. 1b). The results show that the flood maps generated by the simplified specular reflectance model and by the UNIFIED model are very similar. The simulation times for the two models are 1,267 seconds and 777,840 seconds (216.1 hours) respectively. Fig. 13 compares the two flood maps quantitatively in terms of differences in spot positions (centers of the spots) and spot sizes (averaged FWHMs in $\mathrm{x}$ and y direction for each spot). The average and the maximum differences in spot positions (1.3 pixels and 2.3 pixels respectively in the $256 \times$ 
256 pixel flood maps) and sizes (0.1 pixels and 1.9 pixels respectively) are very small. The average spot size of the flood map generated using the UNIFIED model (6.5 pixels) is slightly smaller than that generated using the simplified specular reflectance model (6.6 pixels). There are no significant differences between the flood maps generated by two models. The average number of photons getting collected by 4 PMTs are about 714 and 716 for the simplified simulation GATE simulation respectively.

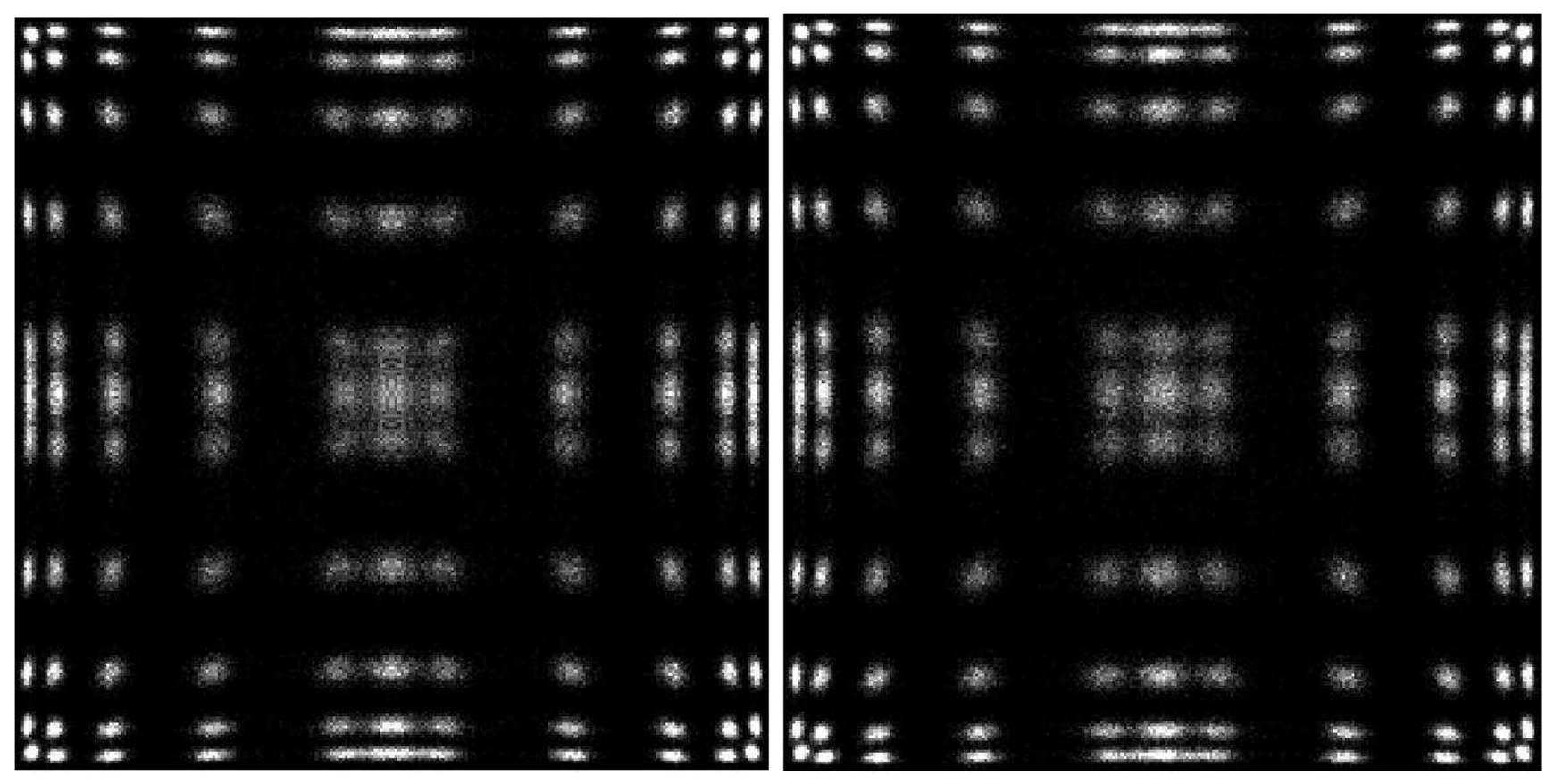

(a)

(b)

Fig. 12. Flood maps of a $12 \times 12$ detector block coupled with a slotted light guide. The six slot depths $\left(a_{0}, \ldots, a_{5}\right.$ as shown in Fig. 7) are $10 \mathrm{~mm}, 6 \mathrm{~mm}, 3 \mathrm{~mm}, 2 \mathrm{~mm}, 1 \mathrm{~mm}$ and $0.5 \mathrm{~mm}$ respectively. The resolutions of the flood maps are $256 \times 256$ pixels. The two flood maps are generated using (a) the simplified specular reflectance model and (b) the UNIFIED model respectively.

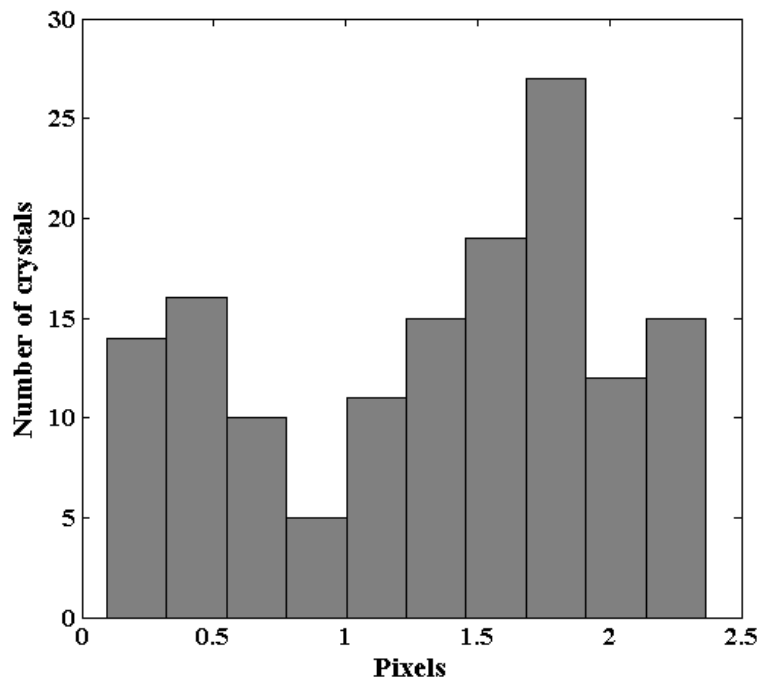

(a)

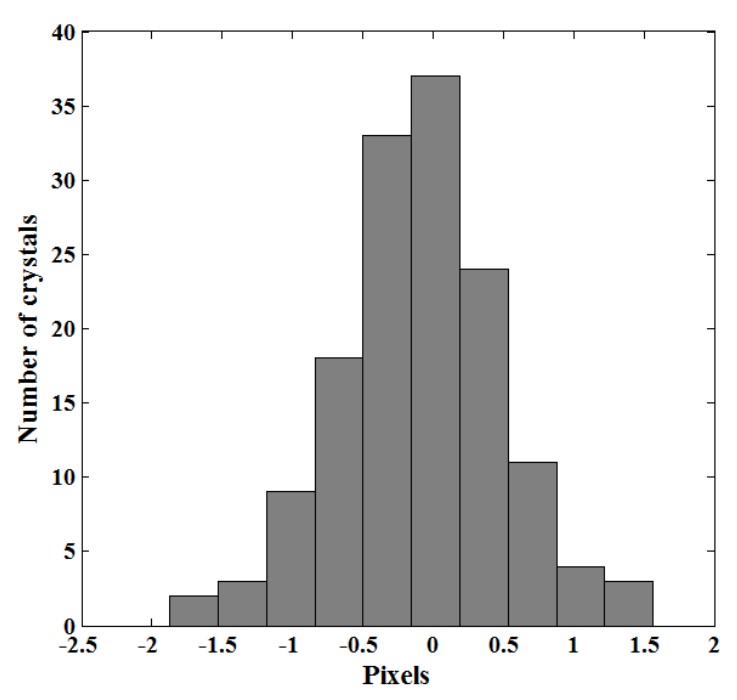

(b)

Fig. 13. The distributions of the differences of (a) the center locations and (b) the FWHMs of the 144 crystal spots of the two flood maps shown in Fig. 12. The average and the maximum differences of the center locations are 1.3 pixels and 2.3 pixels respectively. The average and the maximum differences of the FWHMs are 0.1 pixels and 1.9 pixels respectively. 


\subsection{Flood map optimization}

In Fig. 10, the crystal spots on the edges are almost indistinguishable and the decoding errors for these crystals are therefore very large. In Fig. 12, the crystal spots are mostly distinguishable but are not uniformly distributed. The crystal spots in the middle are more crowded and have larger decoding errors than the other spots. Using the method in Section 2.2.6, we are able to achieve a more uniform flood map by optimizing the slot depths. Fig. 14 shows the flood maps with the optimized slot depths. The six slot depths $\left(a_{0}, \ldots, a_{5}\right.$ as shown in Fig. 7) are $8.1 \mathrm{~mm}, 3.9 \mathrm{~mm}, 2.4 \mathrm{~mm}, 1.3 \mathrm{~mm}, 0.6 \mathrm{~mm}$ and $0.4 \mathrm{~mm}$ respectively, and the spot distribution in Fig. 14 is much more uniform than those in Fig. 10 and Fig. 12. Again, there are no significant differences between the flood maps generated by two models. The simulation times for the two models are 1,253 seconds and 761,184 seconds (211.4 hours) respectively. The average number of photons getting collected by 4 PMTs are about 730 and 732 for the simplified simulation GATE simulation respectively.

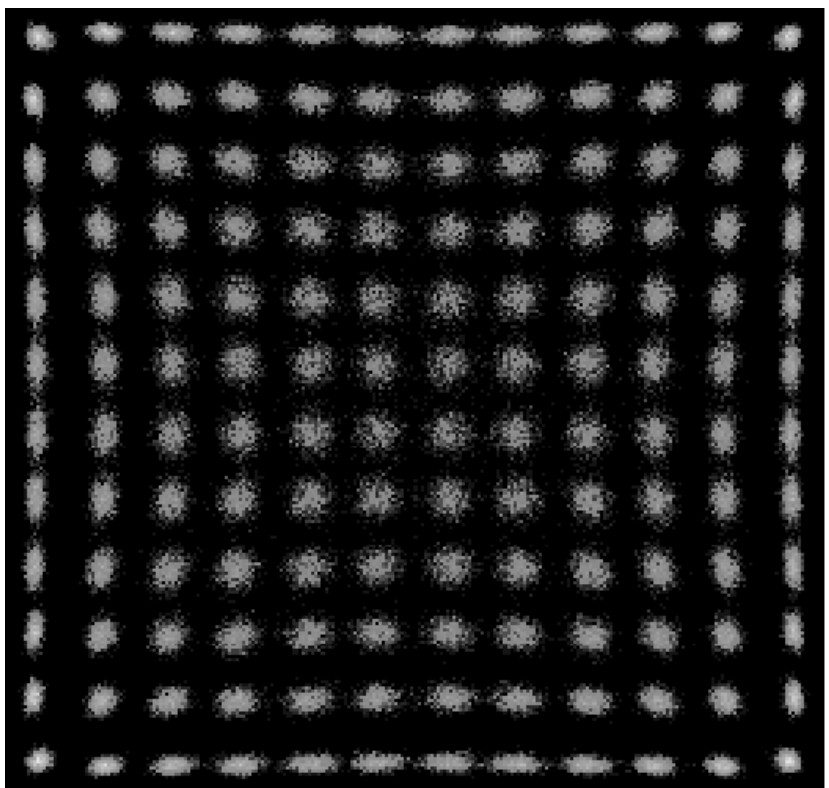

(a)

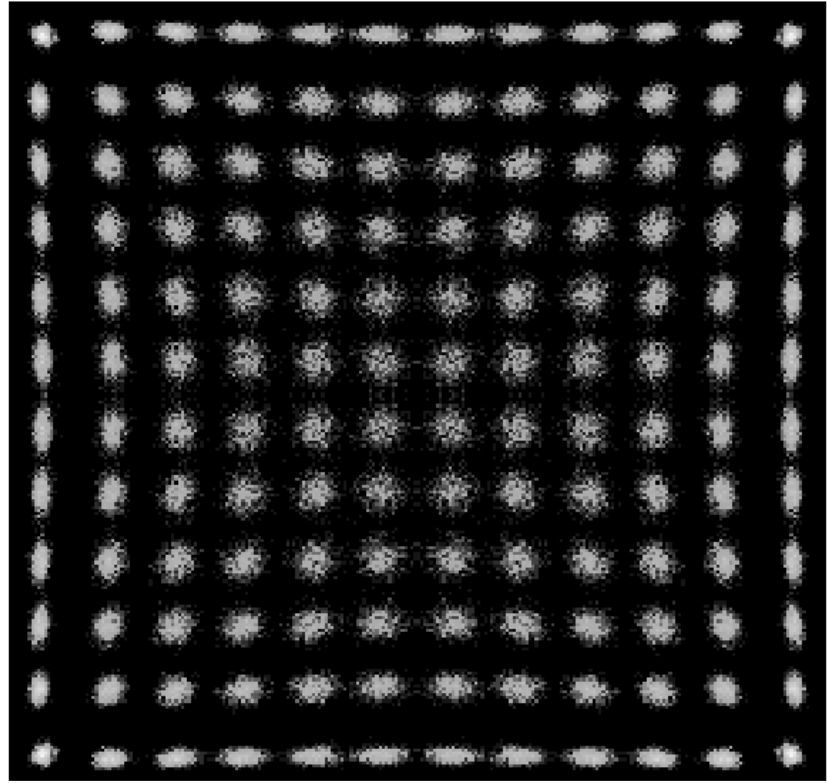

(b)

Fig. 14. Flood maps of a $12 \times 12$ detector block coupled with a slotted light guide with optimized depths. The six slot depths $\left(a_{0}, \ldots, a_{5}\right.$ as shown in Fig. 7) are $8.1 \mathrm{~mm}, 3.9 \mathrm{~mm}, 2.4 \mathrm{~mm}, 1.3 \mathrm{~mm}, 0.6 \mathrm{~mm}$ and $0.4 \mathrm{~mm}$ respectively. The resolutions of the flood maps are $256 \times 256$ pixels. The two flood maps are generated using (a) the simplified specular reflectance model and (b) the UNIFIED model respectively. The average and the maximum differences of the spot center locations between the two flood maps are 1.2 pixels and 2.1 pixels respectively. The average and the maximum differences of the FWHMs are 0.02 pixels and 1.8 pixels respectively. There are no significant differences between the flood maps generated by two models.

Fig. 15 compares the flood maps of detector modules without slots (Fig. 10), with non-optimized slots (Fig. 12) and with optimized slots (Fig. 14). The spot uniformity and shape are evaluated in terms of the distributions of distances between adjacent spots and the FWHMs of spots. Fig. 15a shows that the detector module with optimized slot depths has a much more uniform spot distribution than the module without slots 
and the module with non-optimized slots. Fig. 15b shows that the FWHMs of spots of the two detector modules with slots are slightly smaller than those of the module without slots. That is because the slots in the light guide concentrate the light.

The ratio of the distances to the FWHMs is an indicator of the individual crystal decoding error. The higher the ratio, the lower the decoding error. Fig. 15c shows that all crystals in the detector module with optimized slots have a ratio higher than 1.9. That indicates all the crystals in the optimized module have low decoding errors. In the detector module without slots and the detector module with non-optimized slot depths, some crystals have ratios as small as 0.3 and 0.5 respectively. Those crystals have large decoding errors.

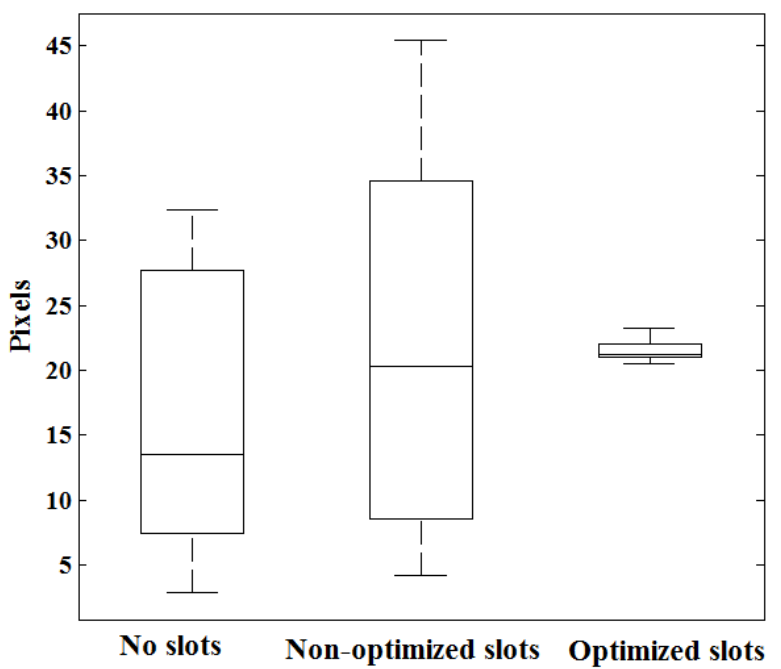

(a)

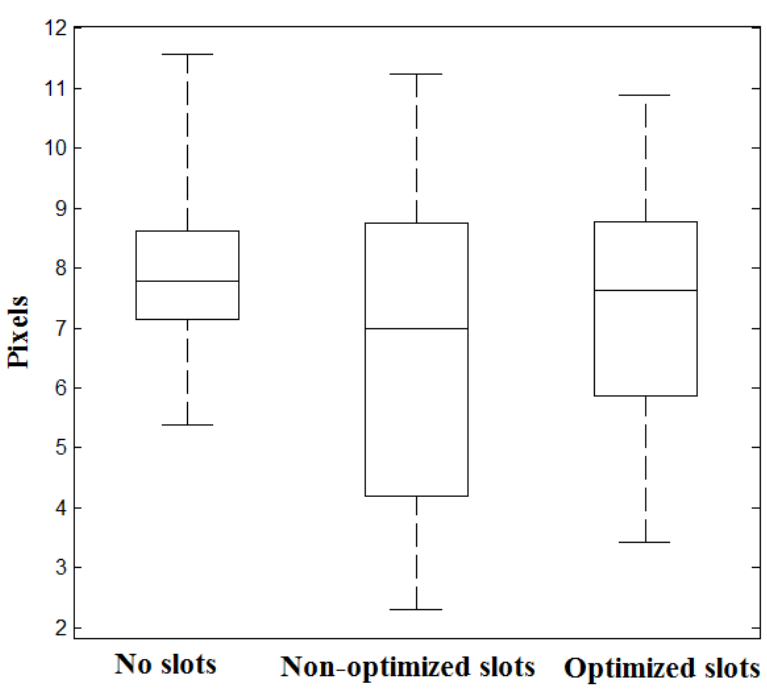

(b)

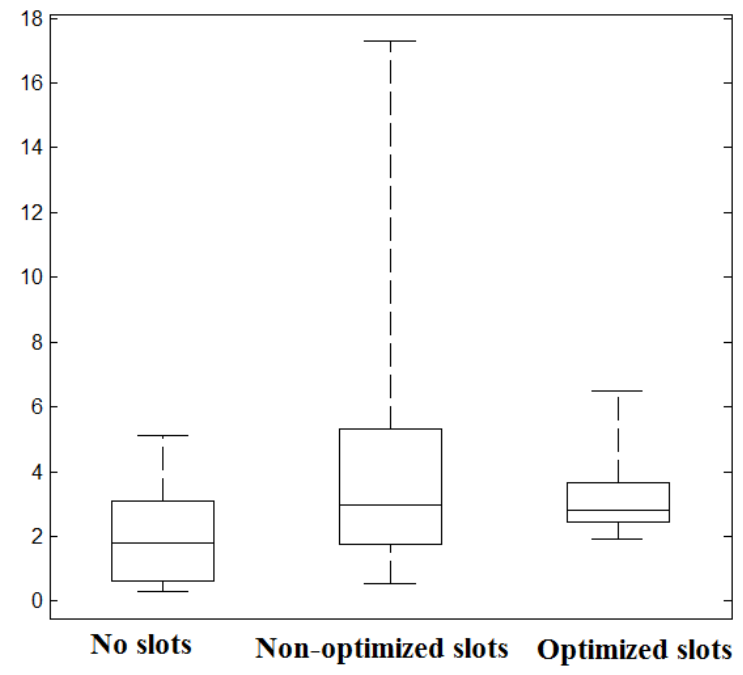

(c)

Fig. 15. (a) Boxplots of the distances between adjacent spots. In the detector module without slots, the Mean \pm SD, minimum and maximum distances are $16.1 \pm 9.9$ pixels, 2.9 pixels and 32.4 pixels respectively. In the detector module with non-optimized slots, the Mean \pm SD, minimum and maximum distances are $23.4 \pm 13.2$ pixels, 4.1 pixels and 45.4 pixels respectively. In the detector module with optimized slots, the Mean \pm SD, minimum and maximum distances are $21.5 \pm 0.7$ pixels, 20.5 pixels and 23.3 pixels 
respectively. (b) Boxplots of the FWHMs of spots. In the detector module without slots, the Mean \pm SD, minimum and maximum FWHMs are $7.9 \pm 1.1$ pixels, 5.4 pixels and 11.6 pixels respectively. In the detector module with non-optimized slots, the Mean \pm $\mathrm{SD}$, minimum and maximum FWHMs are $6.6 \pm 2.5$ pixels, 2.3 pixels and 11.2 pixels respectively. In the detector module with optimized slots, the Mean \pm SD, minimum and maximum FWHMs are $7.3 \pm 2.0$ pixels, 3.4 pixels and 10.9 pixels respectively. (c) Boxplots of the ratio of the distances to the FWHMs between spots. In the detector module without slots, the Mean \pm SD, minimum and maximum ratios are $1.9 \pm 1.3,0.3$ and 5.1 respectively. In the detector module with non-optimized slots, the Mean $\pm \mathrm{SD}$, minimum and maximum ratios are $4.1 \pm 3.4,0.5$ and 17.3 respectively. In the detector module with optimized slots, the Mean \pm SD, minimum and maximum ratios are $3.2 \pm 1.1,1.9$ and 6.5 respectively. The three plots are calculated from the flood maps of the detector modules without slots (Fig. 10), with non-optimized slots (Fig. 12) and with optimized slots (Fig. 14).

Table 2 shows the overall decoding error rates for three detector modules. The module with optimized slots has the lowest ODER. The ODERs calculated from the flood maps generated using the simplified specular reflectance model models are slightly bigger than those using UNIFIED model, but the differences are negligibly small.

Table 2. Overall Decoding Error Rates (ODERs)

\begin{tabular}{|l|l|l|}
\hline & Simplified specular reflectance model & UNIFIED model \\
\hline Without slots & $28.6 \%$ & $28.3 \%$ \\
\hline With non-optimized slots & $10.9 \%$ & $10.7 \%$ \\
\hline With optimized slots & $2.5 \%$ & $2.4 \%$ \\
\hline
\end{tabular}

\subsection{Experimental results}

The same slot depths $(8.1 \mathrm{~mm}, 3.9 \mathrm{~mm}, 2.4 \mathrm{~mm}, 1.3 \mathrm{~mm}, 0.6 \mathrm{~mm}$ and $0.4 \mathrm{~mm}$ respectively) as those simulated in Fig. 14 were used to construct the prototype detector. The simulated flood map and the experimental flood map are compared in Fig. 16. As shown in Fig. 16 b, the quality of the flood map of the prototype detector was excellent. It had nearly uniformly distributed spots similar to those in the simulated flood map (Fig. 16 a). The shapes and orientations of the spots were also very similar to those in the simulated flood map. However, the profile analysis (Fig. 16 c) shows that the flood map generated by simulation and measured from the prototype detector were similar, but not identical. Many factors could have caused the mismatches between the simulation and experimental result. The surfaces of the polished crystals are not perfectly flat and smooth. The effect of the Compton scatters which is not models in our simulation might also have contributed to the mismatch. The insufficient precision of the wire saw we used to machine the slots in the thin metal slices also affected the experimental flood quality. We measured the widths of the slots using a laser confocal microscope and found that they had a variance of about $100 \mu \mathrm{m}$. That led to some slight deformations in the metal slices when the module was assembled. 


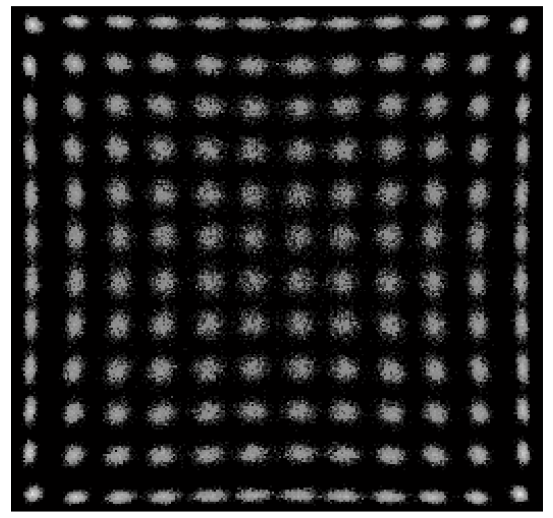

(a)

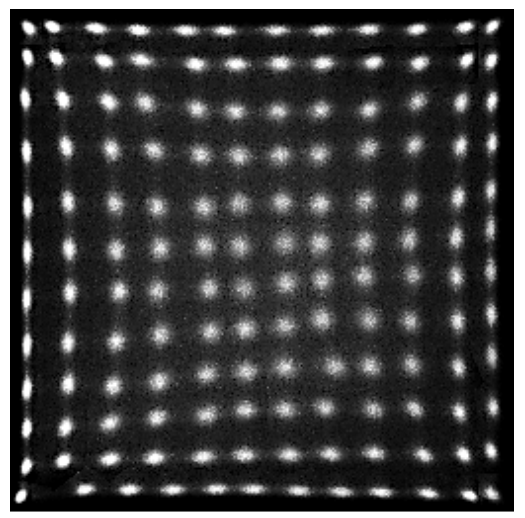

(b)

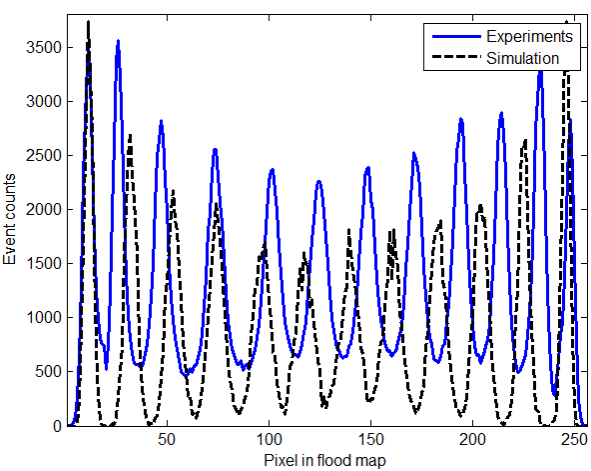

(c)

Fig. 16 (a) Simulated flood map. (b) Flood map of the prototype detector. (c) Profiles of the first column of the two flood maps. The profiles of the prototype detector and simulation result are shown in blue and black, respectively.

\section{Discussion}

In this study we have developed a fast and reliable optical simulation method that is computationally efficient enough to meet the goals of optical simulation. While it is limited to block detector modules constructed with a polished surface finish and using specular reflector materials such as ESR film, many PET detector modules have been constructed using these parameters. We have achieved this using a simplified specular reflectance model and a structured light-tracking algorithm to simulate block detector modules composed of rectangular parallel piped elements. The box-shaped specular reflectance model dramatically simplified the light-tracking computation, and reduced the simulation time. We simulated the same detectors with both the simplified specular reflectance model and the UNIFIED model using a desktop computer with a $3.1 \mathrm{GHz}$ processor. When simulating the detector module without slots in the light guide, the fast approach is 207 times faster than the GATE toolkit. When simulating the detector module with slots in the light guide, the fast approach is more than 600 times faster than the GATE toolkit. It took the fast approach 20 minutes to generate a flood map with one million events, and it took the GATE toolkit 9 days to perform the same task.

The simplified specular reflectance model is validated by comparing its light distribution curve and flood maps with those from the UNIFIED model. The commonly used ESR film reflector is selected in the UNIFIED model. The crystal surface is polished with a deviation of reflective angle equal to $0.31^{\circ}$, which is a realistic value according to Janecek's measurements [18]. The light distribution curves and flood maps generated by the two models are almost identical.

In reality, the deviation of reflective angle may vary when different techniques are used to polish the crystal and/or different specular reflector materials are used. Hence, we also simulated a polished surface with a large deviation of reflective angle of $1.3^{\circ}$ using the GATE toolkit. $1.3^{\circ}$ is a fairly large deviation of reflective angle for a polished surface, considering that those of a ground surface and a chemical etched surface measured by Janecek are $2.03^{\circ}$ and $4.76^{\circ}$, respectively [18]. The flood map shown in Fig. 17 (with a 
large deviation of reflective angle: $1.3^{\circ}$ ) still looks similar to the one generated by the simplified specular reflectance model (Fig. 14a, $12 \times 12$ detector block coupled with a slotted light guide with optimized depths). Similarly, we compared Fig. 17 with Fig. 14a quantitatively in terms of differences in spot positions and spot sizes. The average and the maximum differences in spot positions (3.9 pixels and 6.6 pixels respectively) are three times bigger when the deviation of reflective angle increases from $0.31^{\circ}$ to $1.3^{\circ}$, while the average and the maximum differences in spot sizes ( 0.4 pixels and 2.3 pixels respectively) only change slightly. Again, the average spot size of the flood map generated by the UNIFIED model (6.9 pixels) is slightly smaller than that generated by the simplified specular reflectance model (7.3 pixels). The overall decoding error rates increases slightly from $2.4 \%$ to $2.6 \%$ when the deviation of reflective angle increases from $0.31^{\circ}$ to $1.3^{\circ}$. Those may imply that the slot depths optimized based on the simplified specular reflectance model can be applied to block detectors constructed using crystals poorly polished (with large deviations of reflective angle).

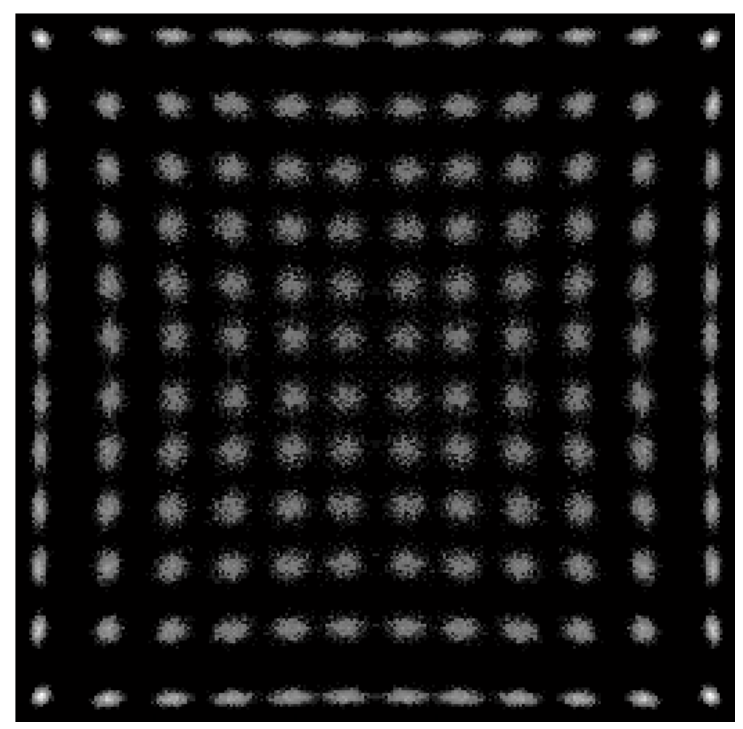

Fig. 17. Flood map generated by GATE with the deviation of reflective angle equal to $1.3^{\circ}$. The slot depths are the same as those used in Fig. 14.

This faster and reliable simulation method allowed us to meet our goal of doing in silico design optimization. The strategy we adapted to improve the flood map is to adjust the slot depths to distribute the spots as uniformly as possible. The simulation results show that the flood map quality could be dramatically improved by applying optimized slot depths. However, flood maps with uniformly distributed spots do not necessarily have fewer crystal decoding errors than those with non-uniformly distributed spots, because the FWHMs of spots are usually not the same. Also, we did not consider practical issues in the simulation, such as the ability to accurately fabricate the slot design that was simulated. Therefore, the slot depths calculated using our strategy are good but not necessarily the best. 
It took our approach 31 minutes to calculate the optimized slot depths for a $12 \times 12$ crystal block detector, and it would take the GATE toolkit more than 14 days to perform the same task. We believe that further performance gains are possible, as the fast algorithm was implemented in Matlab, which is an interpretive language that is slower than the compiled language $(\mathrm{C}++)$ used in GEANT4 and GATE.

\section{Conclusions}

While many Monte Carlo toolkits (including GATE, DETECT and LITRANI) have been developed to simulate the light transport inside scintillation crystals and in complex optical systems such as block detector, they tend to have long computational time, effectively limiting their applications. This could be generally circumvented by parallel computing using multiple processors. However, this problem could be compounded when one wishes to optimize a block detector design in silico, as many block detector design variants must be simulated.

We developed a new approach based on the simplified specular reflectance model and the structured light-tracking algorithm for the rapid optical simulation of light-sharing detector modules. The new approach is 200 to 600 times faster than the GATE simulation with the UNIFIED model, and the two approaches generated comparable flood maps. The new approach has also been validated by constructing a prototype detector and comparing the simulated flood map with the experimental flood map. We conclude that the new approach is a fast and reliable simulation tool for assisting in the development of light-sharing-based detector modules that use a polished surface finish and specular reflector materials.

\section{Acknowledgment}

We thank Dr. Thomas Budinger for helpful discussions and Robert Smith for editing the paper. This work was supported in part by China Scholarship Council (201206210102), International Science \& Technology Cooperation Program of China (2013DFB30270) and Public Health Service grant R01 EB006085, and was carried out at the US Department of Energy Lawrence Berkeley National Laboratory under Contract no. DE-AC02-05CH11231.

\section{References}

[1] C. M. G. Tsang, and J. G. Rogers, "A simulation to model position encoding multicrystal PET detectors," IEEE TRANSACTIONS ON NUCLEAR SCIENCE, pp. 2236-2243, 1995.

[2] S. Surti, J. S. Karp, R. Freifelder, and F. Liu, "Optimizing the performance of a PET detector using discrete GSO crystals on a continuous lightguide," Ieee Transactions on Nuclear Science, vol. 47, pp. 1030-1036, Jun 2000.

[3] E. Roncali and S. R. Cherry, "Simulation of light transport in scintillators based on 3D characterization of crystal surfaces," Physics in Medicine and Biology, vol. 58, pp. 2185-2198, Apr 72013. 
[4] D. J. van der Laan, D. R. Schaart, M. C. Maas, F. J. Beekman, P. Bruyndonckx, and C. W. E. van Eijk, "Optical simulation of monolithic scintillator detectors using GATE/GEANT4," Physics in Medicine and Biology, vol. 55, pp. 1659-1675, Mar 212010.

[5] F. Peng, C. Jing, W. Qingyang, X. Tianpeng, X. Yan, W. Shi, et al., "Optical Simulation of a 9times9 LYSO Block Detector with PQS Technology using GATE," 2012 IEEE Nuclear Science Symposium and Medical Imaging Conference Record (NSS/MIC 2012) \& Workshop on Room-Temperature Semiconductor X-Ray and Gamma-Ray Detectors, pp. 2590-3, 20122012.

[6] H. D. Li, W. H. Wong, S. Kim, S. T. Liu, R. Ramirez, S. P. Xie, et al., "A simulation study on optically decoding reflecting windows for PMT quadrant sharing scintillation detector block," Ieee Transactions on Nuclear Science, vol. 53, pp. 2557-2562, Oct 2006.

[7] D. A. B. Bonifacio, N. Belcari, S. Moehrs, M. Moralles, V. Rosso, S. Vecchio, et al., "A Time Efficient Optical Model for GATE Simulation of a LYSO Scintillation Matrix Used in PET Applications," Ieee Transactions on Nuclear Science, vol. 57, pp. 2483-2489, Oct 2010.

[8] Matthew A. Blackston, F. Habte, Paul A. Hausladen, "A comparison of GEANT4 and DETECT2000 for the simulation of light transport in scintillation detectors," Nuclear Science Symposium Conference Record, 2008. NSS'08. IEEE, pp.4995-4998, 19-25 Oct. 2008, doi: 10.1109/NSSMIC.2008.4774362.

[9] G. C. GATE User's Guide V7, Nov. 2014.

[10] C. Moisan, D. Vozza, and M. Loope, "Simulating the performances of an LSO based position encoding detector for PET," Ieee Transactions on Nuclear Science, vol. 44, pp. 2450-2458, Dec 1997.

[11] H. Rothfuss, M. Casey, M. Conti, N. Doshi, L. Eriksson, and M. Schmand, "Monte Carlo simulation study of LSO crystals," Ieee Transactions on Nuclear Science, vol. 51, pp. 770-774, Jun 2004.

[12] A. Szlavecz, T. Bukki, C. Steinbach, and B. Benyo, "A novel model-based PET detector block simulation approach," Biomedical Signal Processing and Control, vol. 6, pp. 27-33, Jan 2011.

[13] F. Bauer, J. Corbeil, M. Schmand, and D. Henseler, "Measurements and Ray-Tracing Simulations of Light Spread in LSO Crystals," Ieee Transactions on Nuclear Science, vol. 56, pp. 2566-2573, Oct 2009.

[14] Janecek, M.; Wu, h.; Tai. Y.-C., "Design of a Novel Insert PET System for Breast Cancer Imaging”, The Journal of Nuclear Medicine Abstract Book Supplement, vol. 46, no. 5, pp. 209P, May 2005.

[15] W. Wai-Hoi, J. Uribe, K. Hicks, and M. Zambelli, "A 2-dimensional detector decoding study on BGO arrays with quadrant sharing photomultipliers," IEEE Transactions on Nuclear Science, vol. 41, pp. 1453-7, Aug. 1994.

[16] M. Janecek, "Reflectivity Spectra for Commonly Used Reflectors," Ieee Transactions on Nuclear Science, vol. 59, pp. 490-497, Jun 2012.

[17] M. Janecek and W. W. Moses, "Optical Reflectance Measurements for Commonly Used Reflectors," Ieee Transactions on Nuclear Science, vol. 55, pp. 2432-2437, Aug 2008.

[18] M. Janecek and W. W. Moses, "Simulating Scintillator Light Collection Using Measured Optical Reflectance," Ieee Transactions on Nuclear Science, vol. 57, pp. 964-970, Jun 2010.

[19] A. J. Chaudhari, A. A. Joshi, S. L. Bowen, R. M. Leahy, S. R. Cherry, and R. D. Badawi, "Crystal identification in positron emission tomography using nonrigid registration to a Fourier-based template," Physics in Medicine and Biology, vol. 53, pp. 5011-5027, Sep 212008.

[20] H. Du and K. Burr, "An Algorithm for Automatic Flood Histogram Segmentation for a PET Detector," in 2012 Ieee Nuclear Science Symposium and Medical Imaging Conference Record, B. Yu, Ed., ed, 2012, pp. 3488-3492.

[21] C. O. Steinbach, G. Erdei, F. Ujhelyi, P. Major, and E. Lorincz, "Optimized Light Sharing Module for High Resolution PET Block Detectors," Ieee Transactions on Nuclear Science, vol. 59, pp. 507-512, Jun 2012.

[22] H. Peng, P. D. Olcott, V. Spanoudaki, and C. S. Levin, "Investigation of a clinical PET detector module design that employs large-area avalanche photodetectors," Physics in Medicine and Biology, vol. 56, pp. 3603-3627, Jun 212011.

[23] I. Vilardi, A. Braem, E. Chesi, F. Ciocia, N. Colonna, F. Corsi, et al., "Optimization of the effective light attenuation length of YAP : Ce and LYSO : Ce crystals for a novel geometrical PET concept," Nuclear Instruments \& Methods in Physics Research Section a-Accelerators Spectrometers Detectors and Associated Equipment, vol. 564, pp. 506-514, Aug 12006. 